\title{
Biostatistical Analysis on the Combination Treatment Against Acne
}

\author{
Bin Zhao ${ }^{1 *}$, Xia Jiang², Jinming $\mathrm{Cao}^{3}$ and Kuiyun Huang ${ }^{1}$ \\ ${ }^{1}$ School of Science, Hubei University of Technology, China \\ ${ }^{2}$ Hubei University of Technology, Wuhan, Hubei, China \\ ${ }^{3}$ School of Information and Mathematics, Yangtze University, China
}

*Corresponding author: Bin Zhao, School of Science, Hubei University of Technology, Wuhan, Hubei, China.

Received Date: July 09, 2019

Published Date: July 15, 2019

\begin{abstract}
Introduction: Acne is one of the commonest skin disorders. During Adolescence $70 \%$ to $80 \%$ of the population have some clinically evident acne [1]. This is commonly seen as oily face, obstructive horny plugs or inflammatory lesions like pustules. These may complicate into cysts, abscesses, scars, keloids, hyper-pigmentation and above all psychological damage to self-confidence. The striking feature of acne is greasy skin and severity of acne is directly proportional to sebum excretion rate SER [2]. Raised SER is often due to hyper sensitive end-organs i.e. Sebaceous glands (SG), mostly at normal levels of circulating androgens [3]. SG are larger and more permeable in acne skin [4]. Chemabrasion may be introduced as a surgical procedure with a new idea where microdermabrasion (MD) is combined with chemical peeling to achieve permanent partial destruction or shrinkage of target organs i.e. SG. This results in decreased number and size of SG. The MD produces controlled removal of superficial skin layers in successive sessions and the chemical peeler absorbed through abraded opened follicles selectively causes partial destruction of SG. This can be proved by measuring the SER before and after treatment [5-11]. So investigations of SER are very important for understanding the physiology of SG, pathogenesis of acne and the efficacy of this treatment 'chemabrasion sheikhing'.
\end{abstract}

Methods: Every patient had 4 chemabrasion sessions for active acne and for those having scarring 2 additional sessions were done. Sessions were spaced 10 to 15 days. During each session MD was done followed by IMMEDIATE chemical peeling. MD was performed with crystal peel and vacuum system using aluminum oxide crystals. It was almost painless with complete evacuation of comedones and pustules. The abraded area was cooled with ice packs and chemical peel applied. For this study we used a medium strength chemical peeler jessner's solution (JS), created by Max Jessner (having lactic acid, salicylic acid and resorcinol). Burning was controlled with cold saline wipes or ice packs. Topical antibiotic prescribed for twice daily use for five days. Most patients healed in 2 to 3 days.

Results: This combination treatment has proved very safe and effective against acne. All patients had remarkable improvement. We noted a decrease in number of new lesions, skin greasiness and open pores. There was an improvement in scars, hyper pigmentation and we also noted a decrease in vellus hair with more smooth skin.

Keywords: Acne; Sebaceous gland; Microdermabrasion; Chemical Peel; Chemabrasion; Jessner's solution; Sebum excretion rate

\section{Introduction}

The main objective of chemabrasion is to cause permanent reduction of SG and smoothen the scarred skin without any damage to the skin of face. Infact it is difficult to target SG as they are lying deep in the skin. Neither MD alone can affect the SG nor the chemical peel alone can do so. The crystal MD safely removes the well prepared dried skin of face and leaves the mouths of the follicular canals wide open, so the chemical peel applied on the abraded skin gets easily absorbed into the depth of follicles to target SG and parts of the pilo-sebaceous apparatus.
The Survey of literature describes MD and chemical peel used separately but no exactly similarly study known with this object. Some books described methods near to this include:
a) Savant S.S. Text book of dermatosurgery [5]
b) Padilla RS: Cutaneous surgery [6]
c) Brody H: Mosby year book [7]
d) R. Marks: SKIN THERAPY [8] 
e) Stephen H. Mandy: SKIN THERAPY [9]

Main References which inspired to evolve this technique are from the book 'ACNE' by William J. Cunliffe'

\section{Methods}

A total of 100 acne patients were treated in this study including 13 males and 87 females of 15 years and above (Table 1). A study performa was designed to record patients details, severity of acne and follow-up details.

\section{Inclusion criteria}

I. Willing patients only and otherwise healthy

II. No concomitant infection

III. Those patients who had failure after conventional treatment

IV. All patients within COOK'S grading scale 2-8(10), using standard photographic records.

\section{Exclusion criteria}

- $\quad$ Pregnancy (Risks not known)

- Chloracne

- $\quad$ Patients having contact dermatitis

- $\quad$ Rosacea

- $\quad$ Topical allergy to chemical peeler (Table 1)
Table 1:

\begin{tabular}{|c|c|}
\hline Study Period & Jan 2009 to Jan 2013 \\
\hline Total Number of Patients: 100 & Male 13, Female 87 \\
\hline Age: 16 to $30 \mathrm{Y}$ & Mean age $19 \mathrm{Y}$ \\
\hline Duration of illness: 1 to $10 \mathrm{Y}$ & Mean duration: $3.5 \mathrm{Y}$ \\
\hline
\end{tabular}

- $\quad$ All patients had comedones and pustules, 25 had cysts (Cook's grading 2-8).

- $\quad$ All patients gave history of temporary relief with previous treatments but all had recurrence after few weeks.

- All had variable post Inflammatory Pigmentation and scarring. We also noted amount of vellus hair.

\section{Pre-procedure treatment}

* Photos of all patients were taken at the start and during treatment sessions to access the severity of acne and to evaluate the results of chemabrasion.

* All patients prepared with topical retinoid lotion applied twice a day for 5-7 days. This caused intense dryness of face skin which is mandatory for effective abrasion (Figure 1).

* Sensitivity test performed for JS.

* All patients given detailed information about chemabrasion and consent taken.

* No anaesthesia was used as the procedure is almost painless.

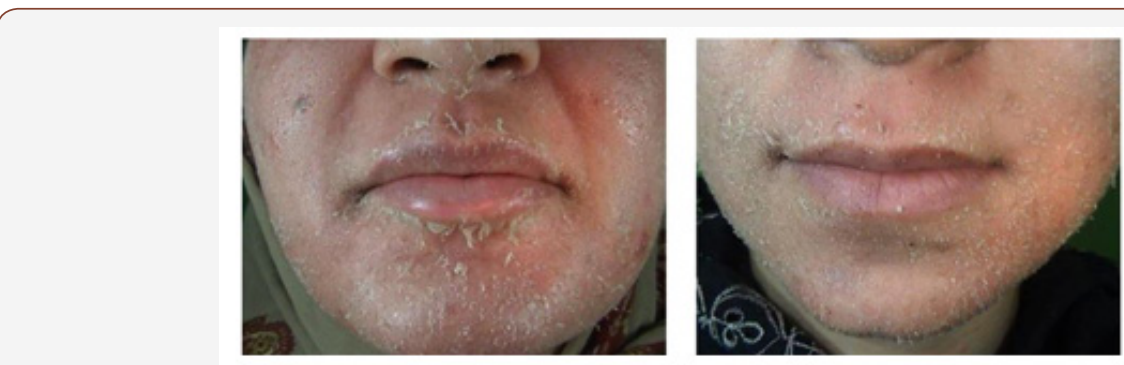

Figure 1: Pre-Procedure preparation with topical retinoid lotion for intense dryness.

\section{Treatment given}

Every patients had 4 chemabrasion sessions for active acne and for those having scarring 2 additional sessions were done. Sessions were spaced 10 to 15 days. During each session MD was done followed by immediate chemical peeling. During each session MD was performed with crystal peel and vacuum system using aluminum oxide crystals. MD was done either as localized disc removal or linear pattern (Figure 2). It was almost painless with complete evacuation of comedones and pustules (Figure 2\&3).
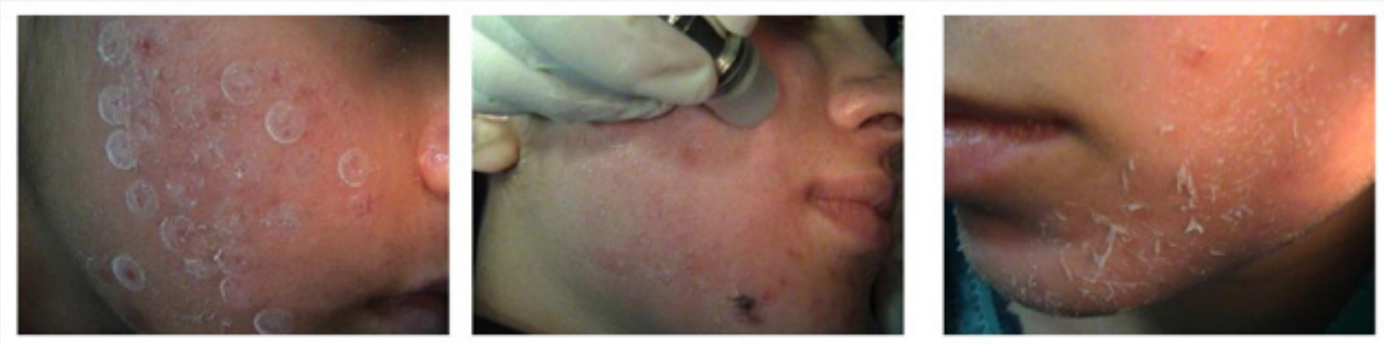

Figure 2: a) MD: thin discs of skin removed over acne and scars. b) Linear MD removingsuperficial skin layers, c) MD: visibly removing skin in flakes and very thin sheets. 

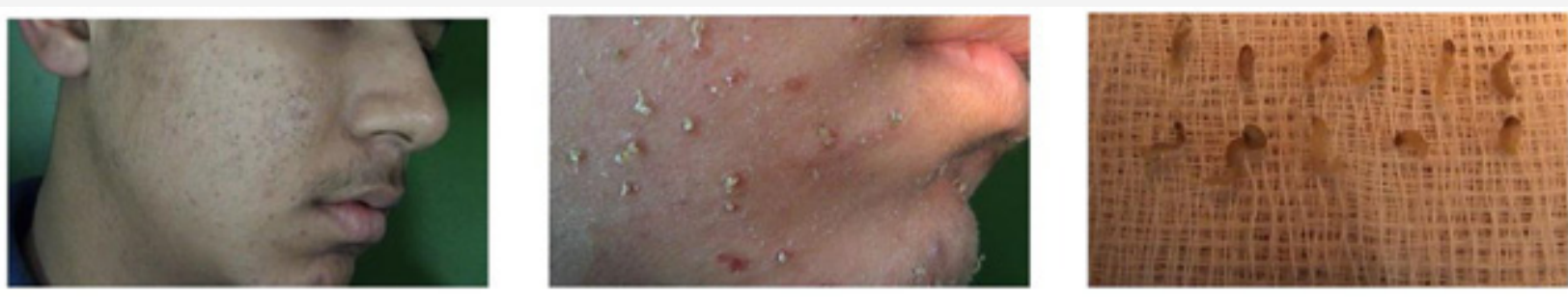

Figure 3: Crystal peel and vacuum system evacuating the waxy horny plugs (the comedones)

Area was cooled with ice pack and chemical peel applied. We used JS as chemical peeler for this study. JS was applied for 30 to 60 seconds till visible frosting (Figure 4). Burning was controlled with

cold saline wipes. Topical antibiotic prescribed for twice daily use. Most patients healed in 2 to 3 days (Figure 4).

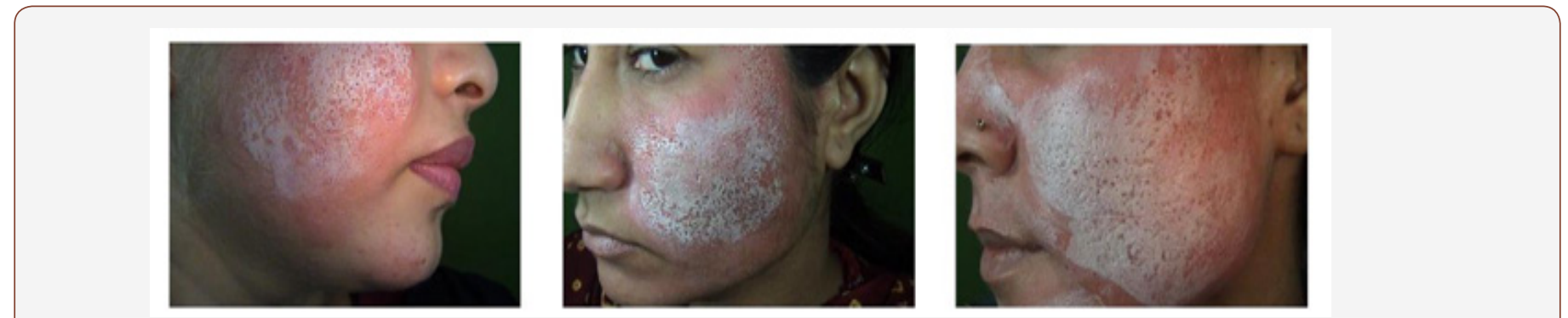

Figure 4: Chemical peeling immediately done on abraded skin till visible frosting. The figures clearly show the JS selectively absorbed through the abraded opened follicular mouths.

\section{Results}

After each treatment session we noted the number of new lesions, greasiness of skin, hyper pigmentation, open pores and scars. To evaluate the results, we suggested a RESULT CRITERIA as following;

$>$ Complete response (80-100\%) no or rare new lesions in next 12 months, no more cysts, obvious decrease in SER.

$>$ Partial response (50-80\%) few small size lesions (4-5 per month), healed fast without pigmentation or scarring, no cysts, SER decreased.
$>$ oNo response $(<50 \%)$ new lesions continued as before, cysts+, SER same.

\section{Our Results}

- $\quad$ Complete Response in 77 Patients (77\%)

- $\quad$ Partial Response in 23 Patients (23\%)

- $\quad$ No Response in 0 Patients $(0 \%)$

All patients had remarkable improvement. It means chemabrasion is a safe and very effective treatment for acne and acne scarring (Figure 5).

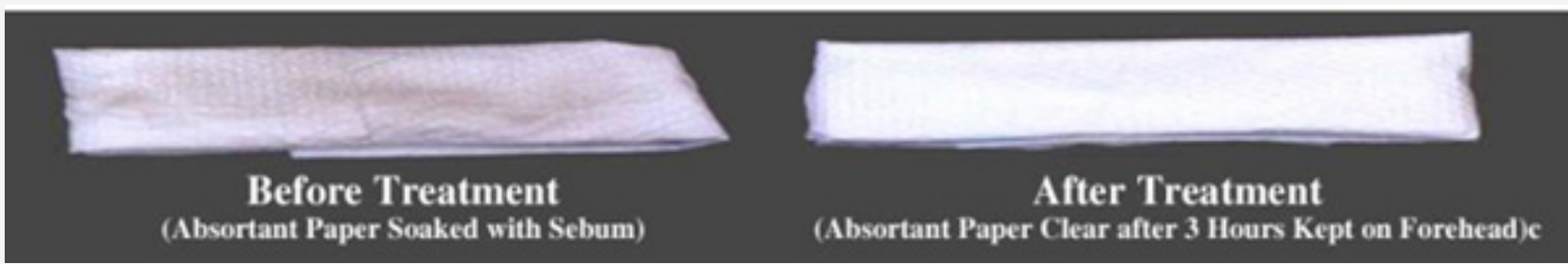

Figure 5: The absorbant paper technique to access the SEP.

\section{Discussion}

How does chemabrasion works?

The main point is that crystal abrasion produces controlled removal of dried-up superficial skin layers in successive sessions and the JS absorbed through opened follicles attacks SG. This can be proved if we measure the SER before and after the treatment [11]. So investigations of SER are very important for understanding the physiology of SG and efficacy of this treatment 'chemabrasion'.

\section{Different methods available to measure the SER are}

- $\quad$ Direct assessment: Surface Microscopy at 50X

- Indirect assessment:

- $\quad$ Lipophilic Stain

- $\quad$ Lipometer

- $\quad$ Planimetry: Analysing SG size (radio labeled, lanthanum)

- $\quad$ Absorbent paper technique 
We used absorbent paper technique [12]. This is random collection of lipid from the skin of forehead, which is SEP (Sebum Excretion and Production) since last washed. Here absorbent paper is applied to the skin of the forehead for a timed period, usually 3 hours and the collected sebum is accessed by oil on absorbant paper (Figure 6). Alternatively the patient's remarks about change in greasy skin are recorded (author's suggestion) (Figure 6).

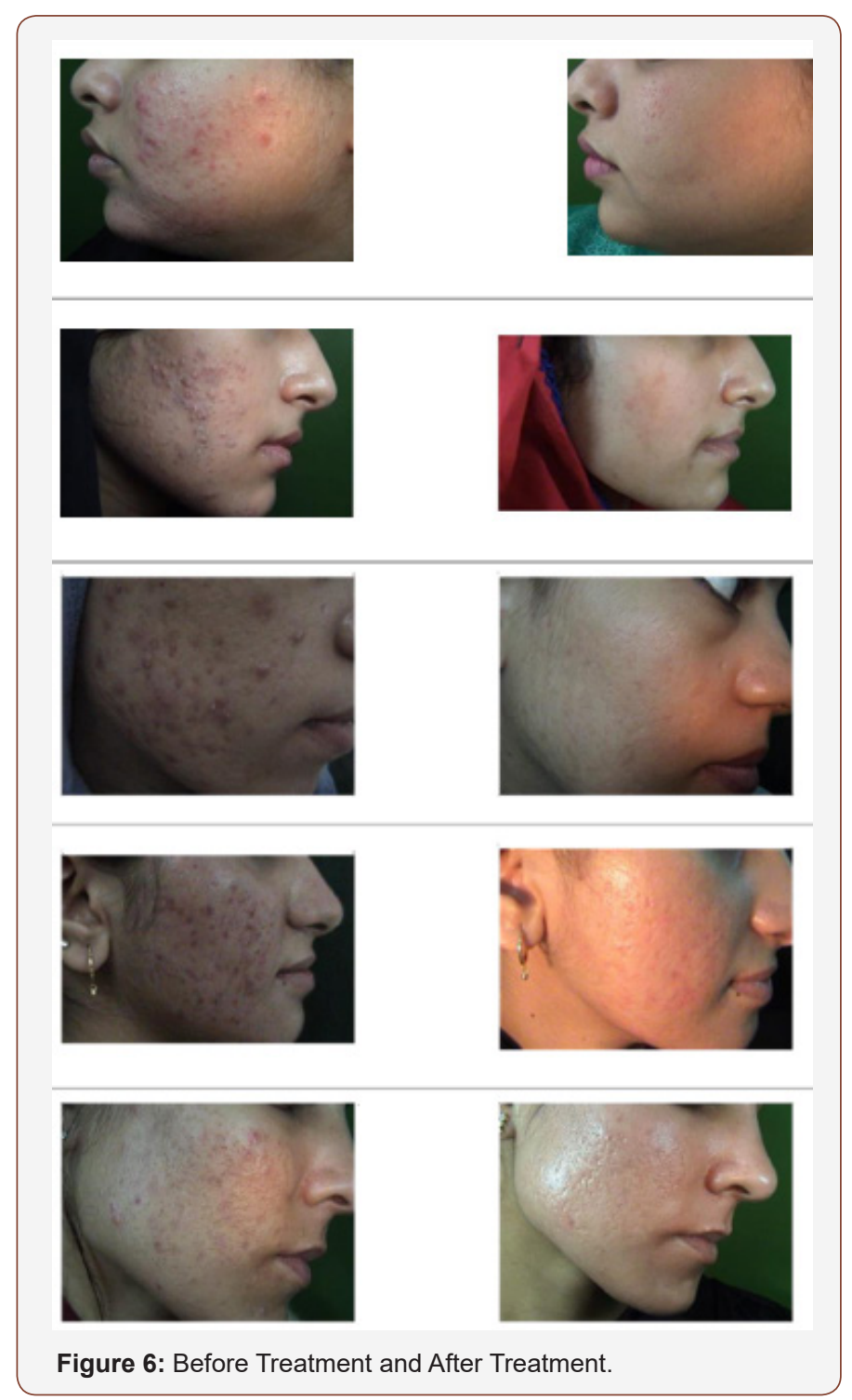

The criticism and further discussion

\section{Positive}

- This method of treating difficult acne is a new hypothesis and original work of the author.

- A good objective as acne is damaging large number of faces causing severe psychological trauma.

- It was done on good number of patient

- The procedure and clinical observations were done by one person only

- $\quad$ Though topical and oral medicines (including antibiotics and retinoids) are effective in good percentage but toxic, costly and recurrence is common. So this alternative treatment approach is available to avoid toxic medication
- Follow-up duration has been long enough 12-18 moths

- All patients filled an evaluation performa and recorded their remarks about the procedure and the results

\section{Negative}

- Proper quantitative investigations of SER needed to prove our observations like:

- Extraction Cup Technique with organic solvent

- $\quad$ Lipid absorbent tape (SEBO TAPE) for timed collection

- $\quad$ Further work needed to have scientific grounds, perhaps a biopsy?

- Perhaps further experience or modification in technique can make it more useful and brief

While performing this study clinical photography was done regularly of all patients. Procedure videos of some patients were made and are available with the author for further reference. Following are photos of some of our acne patients treated during this study with this procedure 'CHEMABRASION SHEIKHING'. The difference in the before and after photos clearly proves the efficacy of this technology (Figure 6).

\section{Conflict of Interest}

There is no conflict of interest and no financial assistance.

\section{Acknowledgement}

This work was supported by the Philosophical and Social Sciences Research Project of Hubei Education Department(19Y049), Hubei Province, China.

\section{References}

1. Bin Zhao (2017) Research on Biomathematics Thought. $1^{\text {st }}$ (Edn.), Science Publishing House, Beijing, China, p. 147.

2. Bin Zhao (2017) Research on Biomathematics Thought. $1^{\text {st }}$ (Edn.), Science Publishing House, Beijing, China, pp. 137-140.

3. Bin Zhao (2017) Research on Biomathematics Thought. $1^{\text {st }}$ (Edn.), Science Publishing House, Beijing, China, p. 250.

4. Bin Zhao (2017) Research on Biomathematics Thought. $1^{\text {st }}$ (Edn.), Science Publishing House, Beijing, China, p. 102.

5. Bin Zhao (2017) Research on Biomathematics Thought. $1^{\text {st }}$ (Edn.), Science Publishing House, Beijing, China, pp. 366-371.

6. Bin Zhao (2017) Research on Biomathematics Thought. $1^{\text {st }}($ Edn.), Science Publishing House, Beijing, China, p. 253.

7. Bin Zhao (2017) Research on Biomathematics Thought. $1^{\text {st }}$ (Edn.), Science Publishing House, Beijing, China, p. 323.

8. Bin Zhao (2017) Research on Biomathematics Thought. $1^{\text {st }}$ (Edn.), Science Publishing House, Beijing, China, p. 296.

9. Bin Zhao (2017) Research on Biomathematics Thought. $1^{\text {st }}$ (Edn.), Science Publishing House, Beijing, China, p. 373.

10. Bin Zhao (2017) Research on Biomathematics Thought. $1^{\text {st }}$ (Edn.), Science Publishing House, Beijing, China, p. 118.

11. Bin Zhao (2017) Research on Biomathematics Thought. $1^{\text {st }}$ (Edn.), Science Publishing House, Beijing, China, p. 123.

12. Bin Zhao (2017) Research on Biomathematics Thought. $1^{\text {st }}$ (Edn.), Science Publishing House, Beijing, China, p. 192. 